\title{
A Wideband Planar Aperture Array Using Interconnected Crossed Rings
}

DOI:

10.1109/TAP.2018.2882612

\section{Document Version}

Accepted author manuscript

Link to publication record in Manchester Research Explorer

\section{Citation for published version (APA):}

Zhang, Y., \& Brown, A. K. (2019). A Wideband Planar Aperture Array Using Interconnected Crossed Rings. IEEE Transactions on Antennas and Propagation, 67(2), 945-950. https://doi.org/10.1109/TAP.2018.2882612

\section{Published in:}

IEEE Transactions on Antennas and Propagation

\section{Citing this paper}

Please note that where the full-text provided on Manchester Research Explorer is the Author Accepted Manuscript or Proof version this may differ from the final Published version. If citing, it is advised that you check and use the publisher's definitive version.

\section{General rights}

Copyright and moral rights for the publications made accessible in the Research Explorer are retained by the authors and/or other copyright owners and it is a condition of accessing publications that users recognise and abide by the legal requirements associated with these rights.

\section{Takedown policy}

If you believe that this document breaches copyright please refer to the University of Manchester's Takedown Procedures [http://man.ac.uk/04Y6Bo] or contact uml.scholarlycommunications@manchester.ac.uk providing relevant details, so we can investigate your claim.

\section{OPEN ACCESS}




\title{
A Wideband Planar Aperture Array Using Interconnected Crossed Rings
}

\author{
Yongwei Zhang ${ }^{\circledR}$, Member, IEEE, and Anthony K. Brown, Life Senior Member, IEEE
}

\begin{abstract}
A 5:1 frequency bandwidth aperture array antenna based on a new planar structure is reported. Dual polarization is implemented with high cross-polarization discrimination over $\pm 45^{\circ}$ scan angle from zenith. It is a low-loss cost-effective approach using minimum dielectric materials. In the proposed planar antenna array design, a mutual coupled interconnected ring structure is used which extends the operational frequency band to the region where the element separation can be considerably greater than half a wavelength, unlike the previously reported interconnected array structures. Hence, the total number of elements can be reduced for a specified aperture size. The dual polarization has coincident phase centers and in the implementation reported here two low-noise amplifiers on the same board are integrated with a pair of dual-polarized elements to form an active array. A 10 by 10 element array prototype of the design has been manufactured and the measured results confirm it as a low cost, high-performance front-end solution.
\end{abstract}

Index Terms-Aperture antennas, phased arrays, planar arrays, radio astronomy.

\section{INTRODUCTION}

W IDEBAND phased array antennas have been becoming increasingly important in a number of applications, often requiring good cross-polarization discrimination and radiation pattern characteristics over wide scan angles. These include radio astronomy [the Square Kilometer Array (SKA)] [1]-[4], satellite-based sub-mm wave instruments, and radar in defense and security [5]. Most solutions reported to date require nonplanar structures such as Vivaldi-type elements with the integration of low-noise amplifiers (LNAs) or transmitter/receiver modules resulting in relatively complex structures. This complexity can be considerable, especially in applications when a large number of elements must be used, e.g., SKA.

Vivaldi arrays are widely reported among a number of wideband arrays, having the potential of 10:1 bandwidth or more but Vivaldi array elements are typically a few wavelengths long at the highest frequency to achieve this bandwidth [6], [7]. Vivaldi-type designs also suffer from high cross-polarization coupling while scanning particularly in the $\pm 45^{\circ}$ planes [8].

Manuscript received January 29, 2018; revised September 16, 2018; accepted October 29, 2018. Date of publication November 21, 2018; date of current version February 5, 2019. This work was supported by STFC U.K. under Project ST/M001482/1. (Corresponding author: Yongwei Zhang.)

The authors are with the School of Electrical and Electronic Engineering, The University of Manchester, Manchester M13 9PL, U.K. (e-mail: david.zhang@manchester.ac.uk).

Color versions of one or more of the figures in this paper are available online at http://ieeexplore.ieee.org.

Digital Object Identifier 10.1109/TAP.2018.2882612
To overcome size and complexity limitations planar structures are attractive. Planar array structures were studied as early as the 1960s. Wheeler [9] was one of the first to use analytical methods to study electronically scanned arrays and proposed the electric current sheet. It was noted by Wheeler [10] that "current sheet" or "continuous current array" demonstrates the real impedance in the absence of a ground plane. However, a ground plane below the current sheet is normally needed to limit the radiation to the upper hemisphere. This will degrade the input impedance over a wide frequency range due to image currents, particularly when scanning in the E-plane.

Munk [11] proposed one approach to realize such current sheet, which consists of closely spaced wires fed periodically. The current sheet array (CSA) developed by Munk et al. [12] is one of the first realizations of Wheeler's current sheet incorporating a ground plane. This is based on the principle that a metallic ground plane next to a CSA can be treated as a simple inductance $\left(Z_{\mathrm{GP}}=j Z_{0} \tan \beta d\right.$, where $d$ is the distance from the array to the ground plane), at the input terminals of the array [13]. By introducing end tip capacitors between the dipole elements, this dominating inductance is largely cancelled and an approximate sinusoidal current distribution is maintained over a wide bandwidth.

Bandwidth limits of such a planar aperture placed above a ground plane were investigated in [14], which introduced the idea of additional multiple dielectric layers on the top of the array structure to provide further bandwidth extension, in addition to a dielectric substrate layer between the array sheet and the ground plane. Complex substrate/superstrate combinations and intensive numerical optimization are needed to achieve a wide bandwidth (the order of array needs to be a large number). Consequently, the substrate and the material loading (several layers of matching superstrates) will potentially reduce the efficiency of the array due to dielectric losses.

In this paper, we will discuss a 2-D antenna array structure which extends the basic concepts of an interconnected dipole array by using crossed octagonal ring elements each element being balanced fed and interconnected to its neighbors, the resulting crossed octagonal ring antenna (C-ORA) provides wideband, dual-polarized performance without requiring a substrate and layers of superstrate slabs [15]. Hence, it can be low-resistive loss and low profile.

Initially, the basic octagonal ring element was proposed using two pairs of mutually capacitive enhanced octagonal rings in an $\mathrm{L}$ configuration with one of the rings shared to provide dual polarization [15], [16]. This can achieve a 5:1 bandwidth by including a single superstrate layer of 
meta-material above the active antenna array surface. The C-ORA reported here uses two pairs of mutually capacitive rings without sharing rings, therefore, coincident phase center feeding for both polarizations can be realized. This paper also reports the optimization of the basic octagonal ring element allowing further bandwidth extension. Both ORA and C-ORA can be scaled to different frequency bands using a few key parameters without requiring intensive numerical optimization. The design and measurements on C-ORA finitearray prototype are reported in this paper.

\section{Crossed Octagonal Ring Antenna}

In phased arrays, the separation between elements is limited by grating lobe requirement, the array element separation is typically between $\lambda / 2$ and $3 / 4 \lambda$ at the highest frequency of operation depending on the maximum scan angle required.

For planar arrays, antenna radiators in most cases need to be above a metallic ground plane to limit radiation below the antenna. It is understood that when the distance between the array antennas and the ground plane is $\lambda / 4$, the ground plane is seen as an open circuit and the input impedance of element is similar to that in free space; at higher frequencies where the distance is greater than $\lambda / 4$, the effect from ground plane becomes capacitive. When the distance approaches $\lambda / 2$, the array tends to be short circuited by the ground plane. The distance to the ground plane, therefore, needs to be less than $\lambda / 2$ at high frequencies to avoid being short-circuited.

In wide bandwidth planar arrays, the main challenge is at low frequencies, where the distance from the array to the ground plane is much less than $\lambda / 4$. At these frequencies, the ground plane leads to a significantly increased inductive reactance for the input impedance of the array antenna. Munk's [11] innovation uses capacitors between the elements to counteract the ground plane inductance at the low frequency. This has some shortcomings, although it shows better performance than a simply connected dipole array. The capacitance introduced between the elements shows frequency dependence and it cannot cancel ground plane inductance completely. At lower frequencies, the capacitance effect starts to dominate, $1 / j \omega C$, i.e., at the extreme case of dc, it makes the array open circuited. However, a low radiation resistance at the low-frequency band still exists, as would be the case in a nonconnected close spaced dipole array.

In very wideband applications, the capacitive effect of ground plane at the high frequency is also of concern. To reduce the total number of elements in a specified aperture size or obtain enough space for proximate electronic integration, a large array element separation is needed, limited only by avoiding grating lobe production. Hence, the array is assumed to operate over a large fraction of the frequency band where the ground plane shows a capacitive effect. Intuitively, this effect appears beneficial in cancelling the inductance effect of the antenna shown at the high frequency. It is known that a dipole antenna tends to be inductive at high frequencies. However, the inductance dominates the total reactance (the capacitive reactance is a combination of the tip
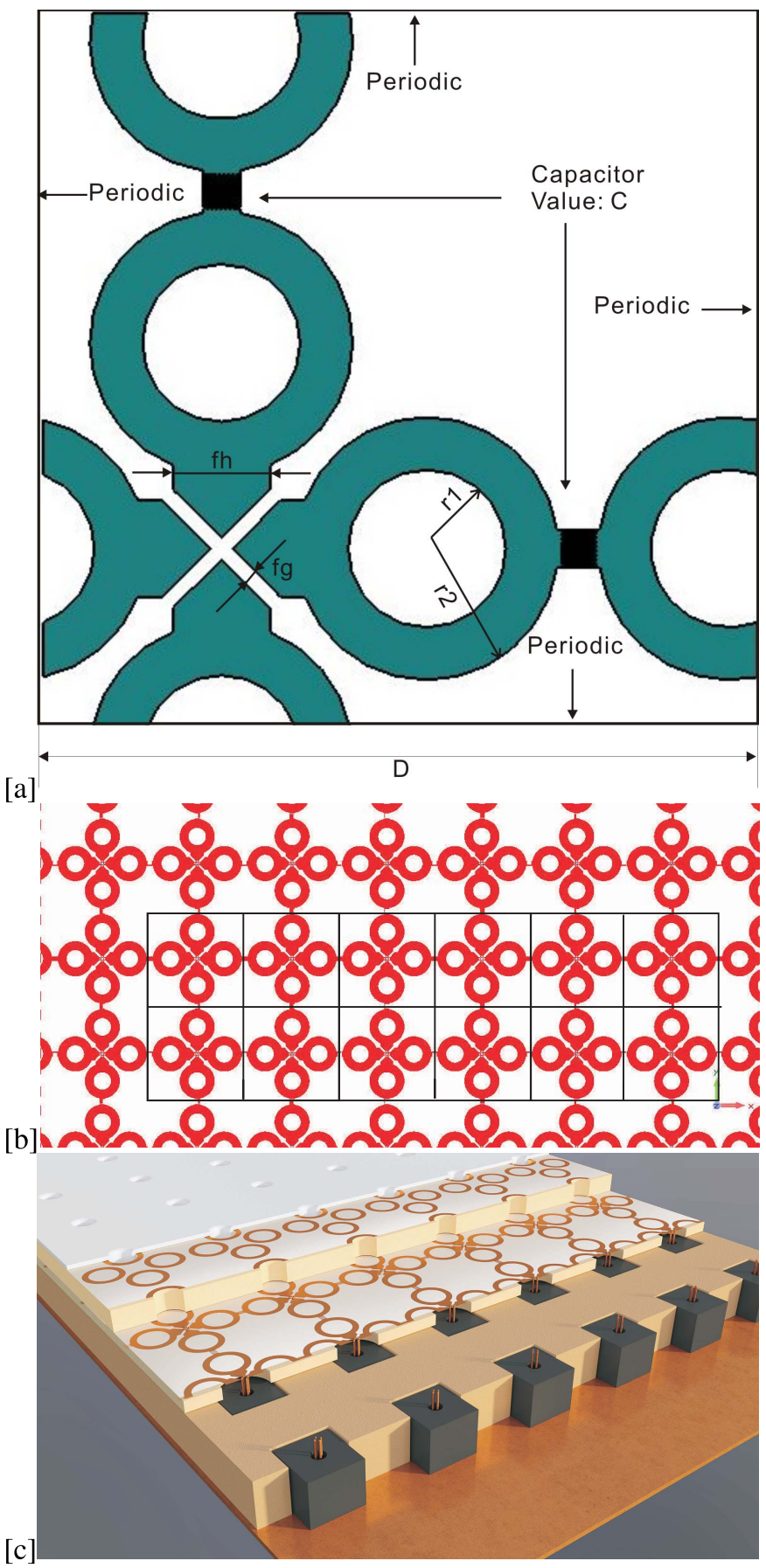

Fig. 1. C-ORA design. (a) Unit cell. (b) Section of the C-ORA array. (c) Layered structures of the C-ORA array.

capacitance mentioned earlier and the capacitance from the ground plane). This is more problematic when the array is scanned, especially for high angle scans in the E-plane, where the array elements tend to be more inductive at high frequency and the input impedance shows a strong inductive reactance if still using the same capacitor.

In addition to the limiting effect of the grating lobes intersection, the dominating inductance at the high frequency is the obstacle to maximizing the element separation and the operation frequency bandwidth. To improve bandwidth/scan performance, a C-ORA is proposed by introducing rings in 

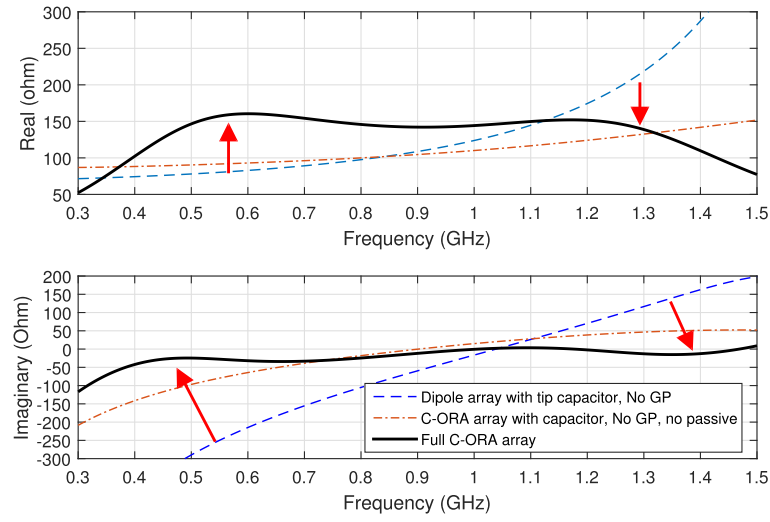

Fig. 2. Ring effect on the array.
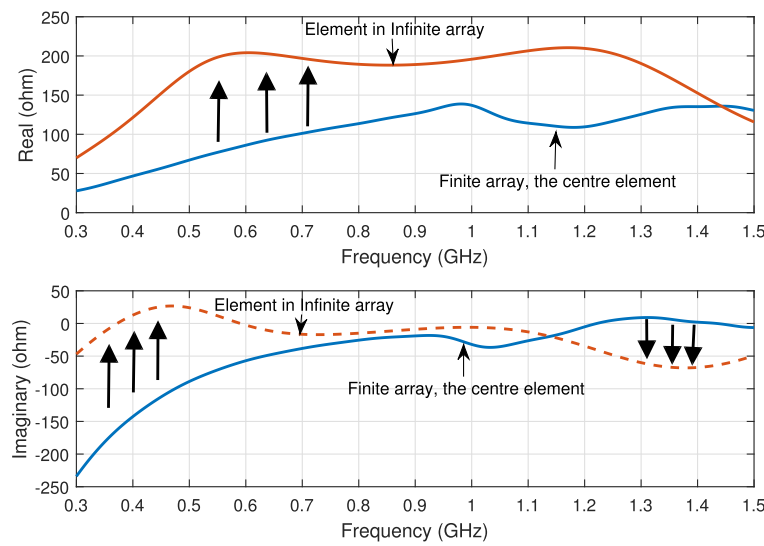

Fig. 3. Input impedance.
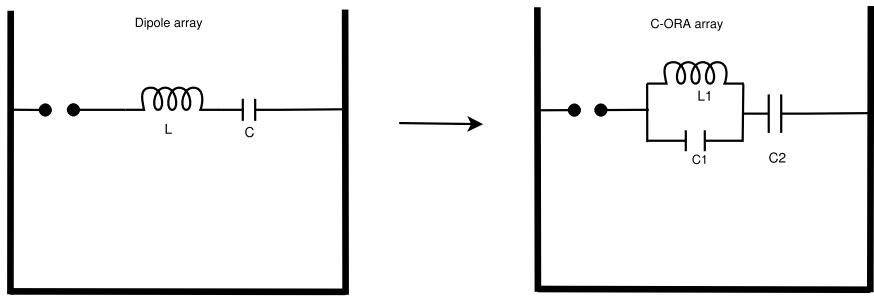

Fig. 4. Equivalent circuits between dipole and C-ORA array.

the radiating arms instead of the conventional dipole structure. This is based on an advancement of the ORA reported in [15]. The crossed ring antenna structure used in the C-ORA is shown in Fig. 1. Both of these two antenna structures use rings as the radiators. The ring structure for C-ORA, however, has been optimized with respect to its self-generated capacitance. This is used to help combat the inductance at the high frequencies resulting in a broader operational bandwidth. Both ORA and C-ORA are formed by two layers: an active layer and a passive layer. The active layer is $\lambda / 4$ above the ground plane at a frequency somewhat below the maximum operational frequency, and the passive layer is half of this distance above the active layer. In this implementation, the separation between the active layer and the ground plane is $\lambda / 4$ at $1.2 \mathrm{GHz}$, where the highest operational frequency of the array is $1.45 \mathrm{GHz}$.

The ring effect can be observed in Fig. 2. Compared with a $2 \mathrm{~mm}$ wide strip planar dipole with tip capacitor of $0.2 \mathrm{pF}$, the C-ORA rings, combined with the optimized capacitance between rings, leads to a low inductive terminal input impedance at the antenna elements even at the highest
TABLE I

PARAMETERS FOR THE C-ORA DESIGN

\begin{tabular}{|c|c|l|}
\hline \multicolumn{3}{|c|}{ C-ORA design } \\
\hline Parameter & Value $(\mathrm{mm})$ & Description \\
\hline $\mathrm{D}$ & 125 & Element separation \\
\hline $\mathrm{r} 1$ & 13.8 & Radius for inner circle \\
\hline $\mathrm{r} 2$ & 23 & Radius for out circle \\
\hline $\mathrm{fh}$ & 17 & Width for feeding strip \\
\hline $\mathrm{fg}$ & 2.83 & Gap for feeding strip \\
\hline
\end{tabular}

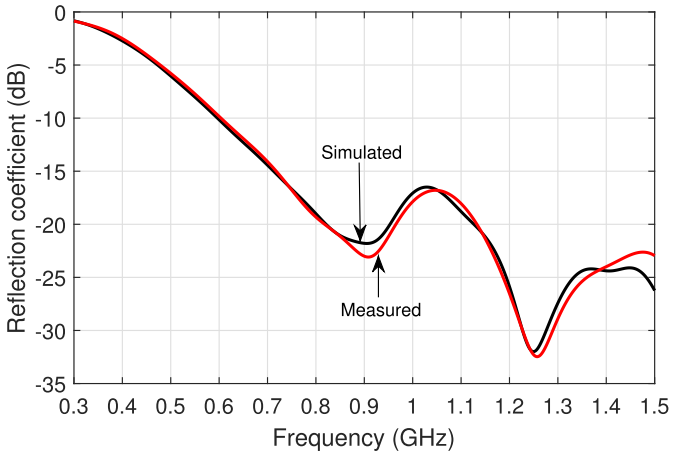

[a]

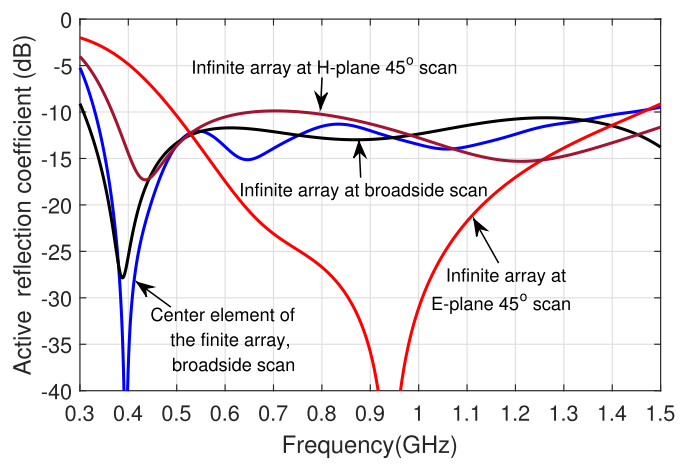

Fig. 5. Reflection coefficients. (a) Center element of the finite array with the surrounding elements terminated with matched loads. (b) Active reflection coefficients of the array elements.

frequency of operation. This capacitive effect of the rings particularly at the high-frequency band becomes more significant for arrays with a greater element separation as bigger rings can be used. As shown in Fig. 3, where the element separation is $125 \mathrm{~mm}$ with the diameter of for the ring, $r_{1}=23 \mathrm{~mm}$, $r_{2}=13.8 \mathrm{~mm}$, more capacitance is observed over the same frequency band than the array with element separation of $105 \mathrm{~mm}$, where the diameter for the ring, $r_{1}=20 \mathrm{~mm}$, $r_{2}=12 \mathrm{~mm}$. The equivalent circuit model for the C-ORA is shown in Fig. 4. A parallel capacitor is introduced along the inductive radiator arm to represent the capacitive effect of the rings.

The input impedance of the elements in the C-ORA is shown in Fig. 3. An optimized model with the element separation of $125 \mathrm{~mm}$ is given in Table I.

\section{Finite ARray Design StUdy}

The C-ORA design model is optimized to cover the frequency range between 450 and $1450 \mathrm{MHz}$. The element separation of $125 \mathrm{~mm}$ is used, which is a half wavelength at $1200 \mathrm{MHz}$. A finite array of $10 \times 10$ elements was manufactured so that the physical aperture of the array is $1.25 \mathrm{~m} \times 1.25 \mathrm{~m}$. As a result, the aperture size is slightly less 
[a]
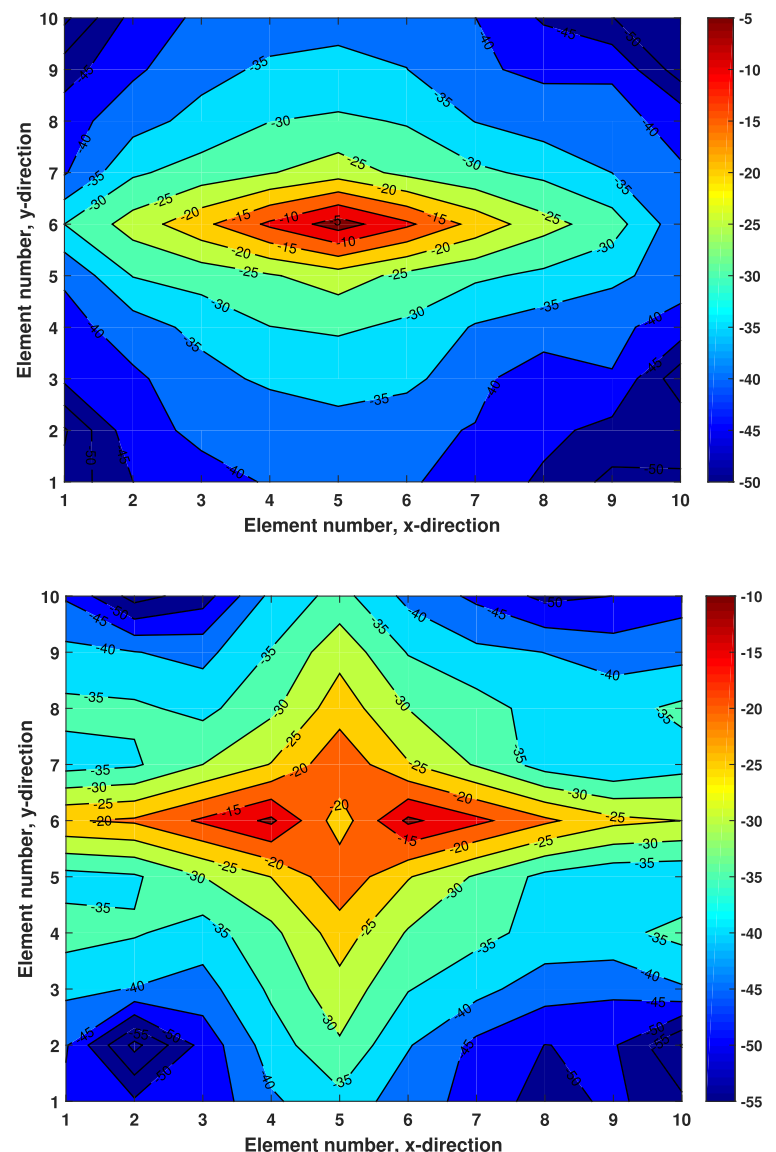

[b]

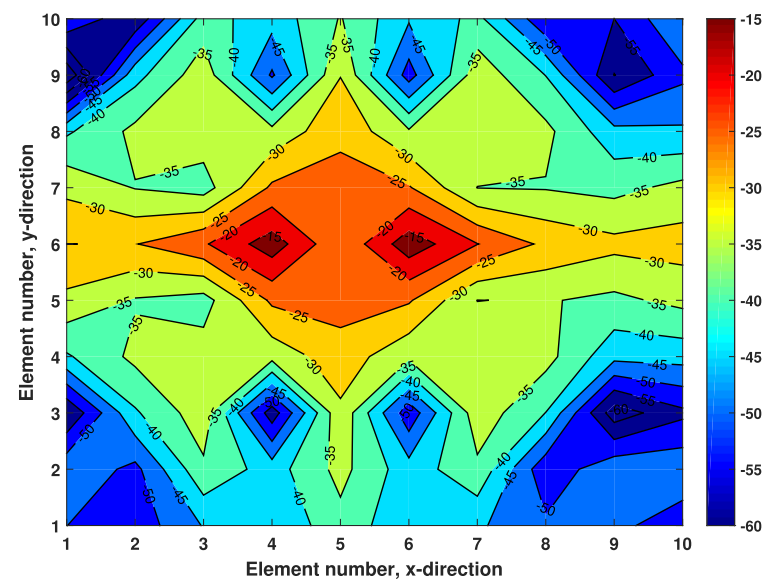

[c]

Fig. 6. Mutual coupling between the center element and the neighboring elements, the center element has the element number 5 in both directions. (a) $400 \mathrm{MHz}$. (b) $900 \mathrm{MHz}$. (c) $1400 \mathrm{MHz}$.

than $2 \lambda$ at the lowest frequency and approximately $6 \lambda$ at the highest frequency. The reflection coefficient of the center element of the finite array when all surrounding elements are terminated with a matched load is shown in Fig. 5(a). The active reflection coefficient of the center element in the finite array is shown in Fig. 5(b), for comparison, the elements in an infinite array with all elements active is also shown. Some minor edge effects from the finite array can be observed over the frequency band, however, the general agreement is good.

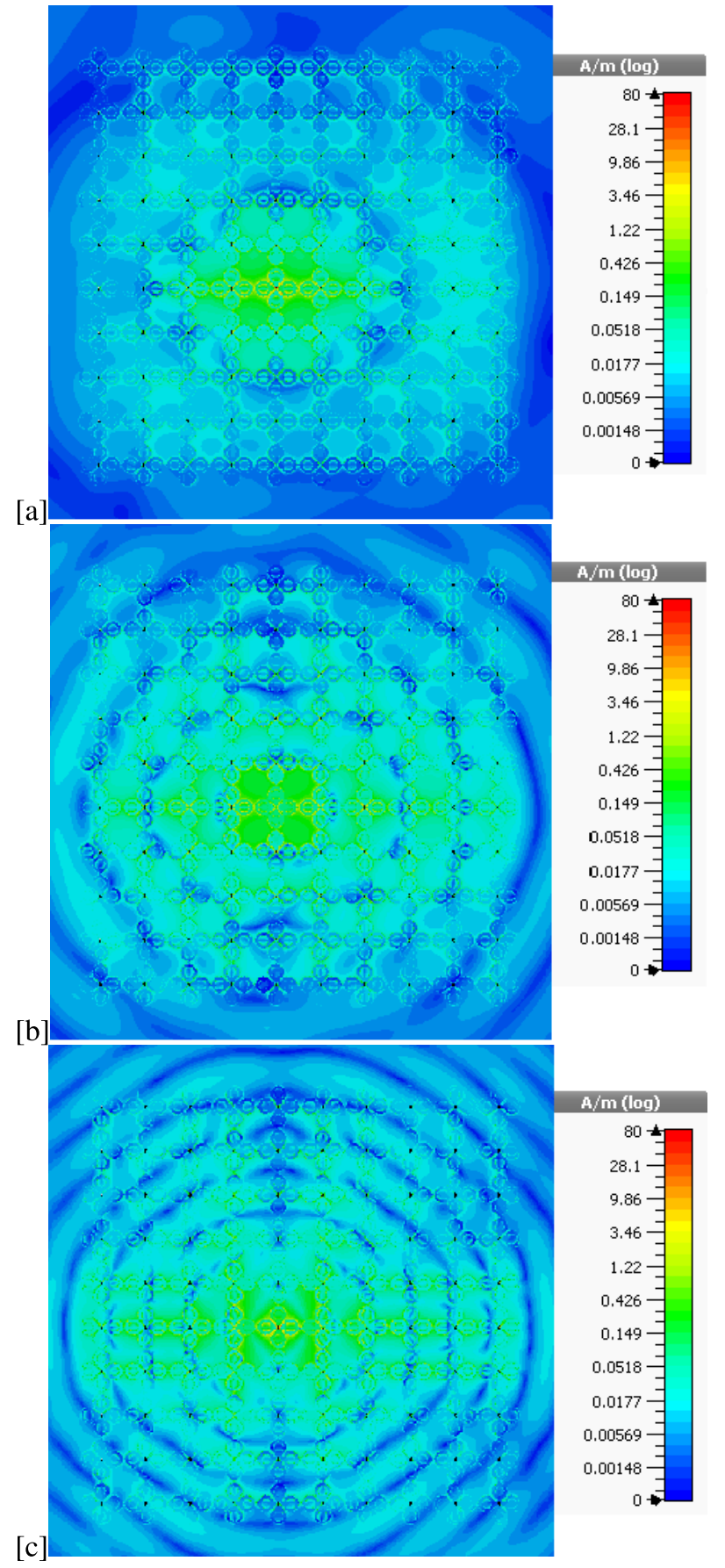

Fig. 7. Simulated surface current from excitation on the center element. (a) $400 \mathrm{MHz}$. (b) $900 \mathrm{MHz}$. (c) $1400 \mathrm{MHz}$.

The mutual coupling between the center element and the surrounding elements is shown in Fig. 6. It indicated that the mutual coupling at the low frequency becomes less significant gradually as the distance increases from the excited element. This is as expected. The coupling at the high frequencies shows a more complex pattern but, in general, coupling between the adjacent elements is much stronger at the low frequency than at high frequency.

The mutual coupling is optimized by controlling the capacitor between the element rings and choosing the size of rings. The aim is to produce the CSA of wide bandwidth. The effectiveness of the C-ORA design to achieve this can be 


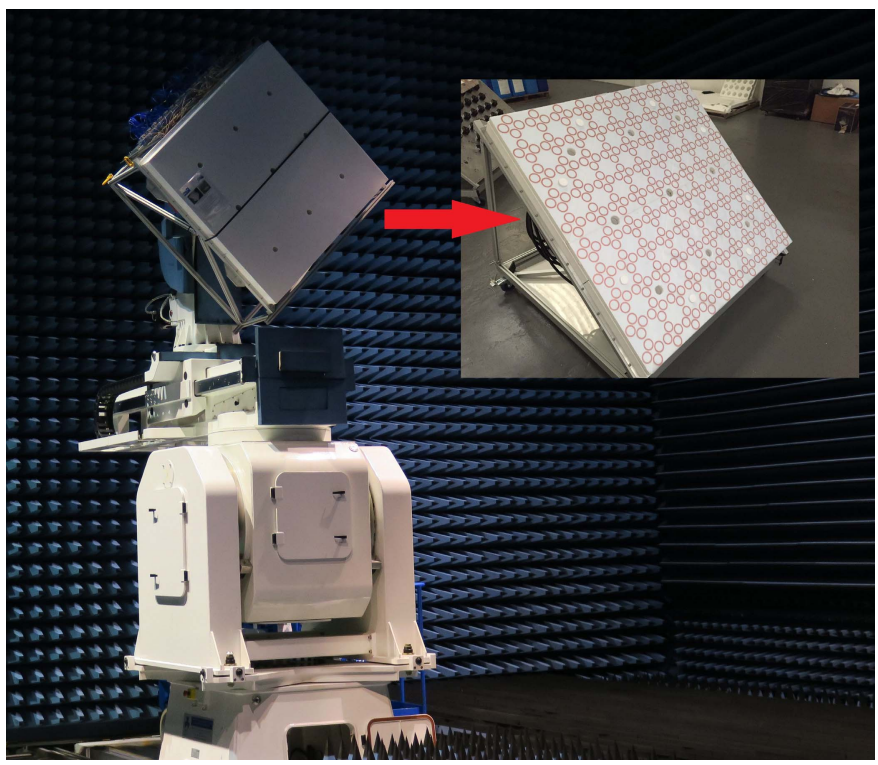

Fig. 8. Finite C-ORA array prototype under test.
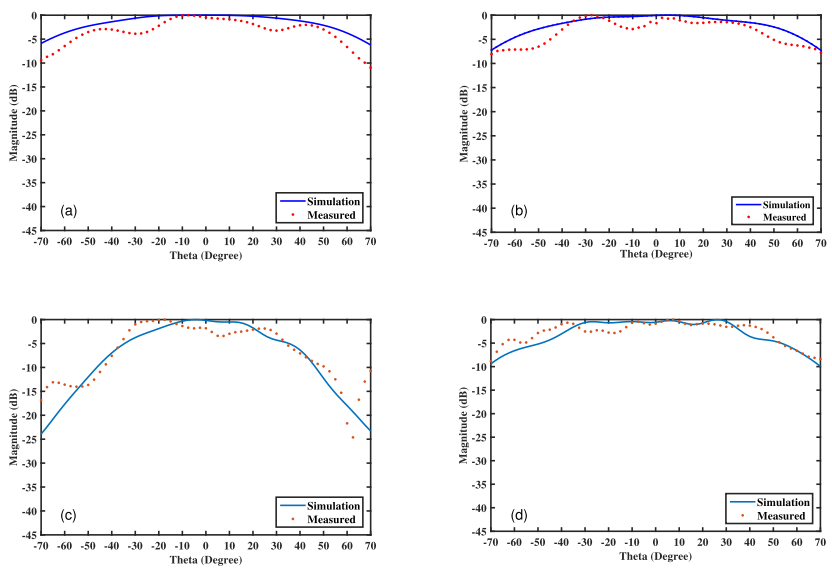

Fig. 9. Measured radiation patterns of the center element in the $10 \times 10$ finite array. (a) E-plane, $900 \mathrm{MHz}$. (b) H-plane, $900 \mathrm{MHz}$. (c) E-plane, $1400 \mathrm{MHz}$. (d) H-plane, $1400 \mathrm{MHz}$.

demonstrated by monitoring the surface current. When the center element in the finite array is excited, with all the other elements terminated with matched loads, the current flows from the active element to the neighboring elements along the array surface. The approximate sinusoidal current flow is maintained across the frequency band as shown in Fig. 7. This distribution of the surface currents at different frequencies confirms the wideband continuous current sheet created by the C-ORA design.

\section{Measurements and Results}

The finite-array prototype has been measured in a compact range. The compact range limits the lowest measurable frequency. The physical aperture size of the finite C-ORA array is $1.25 \mathrm{~m} \times 1.25 \mathrm{~m}$. Directional EMC Test-Antenna HyperLOG 3080 from Aaronia AG is used as the transmit antenna for the C-ORA array radiation pattern measurements. This is shown in Fig. 8. The active layer is made by chemical etching on N9000 PTFE (board thickness $0.127 \mathrm{~mm}, \epsilon_{r}=2.2$, $\tan _{d}=0.0009$, and $17.5 \mu \mathrm{m}$ copper). The passive layer is etched on the polyester sheet with the thickness of $50 \mu \mathrm{m}$.
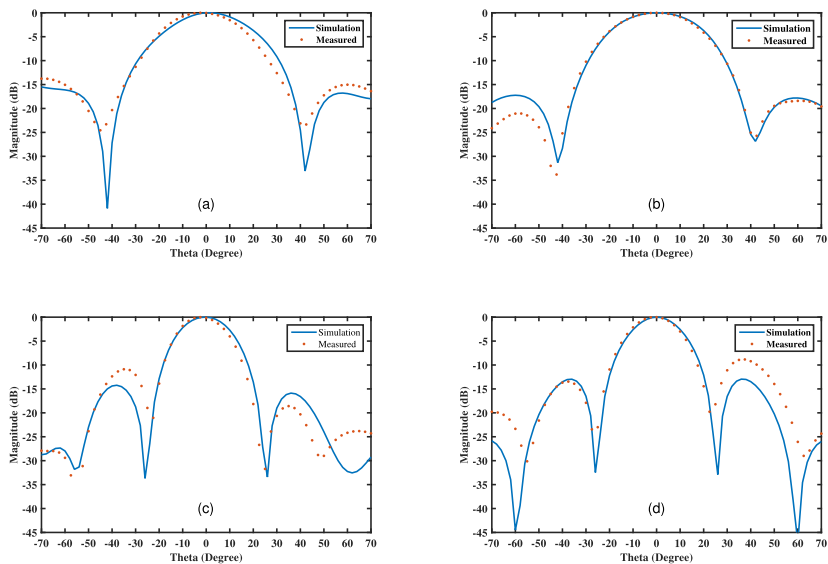

Fig. 10. Measured radiation patterns of the $4 \times 4$ subarray at the boresight scan. (a) E-plane, $900 \mathrm{MHz}$. (b) H-plane, $900 \mathrm{MHz}$. (c) E-plane, $1400 \mathrm{MHz}$ (d) H-plane, $1400 \mathrm{MHz}$.

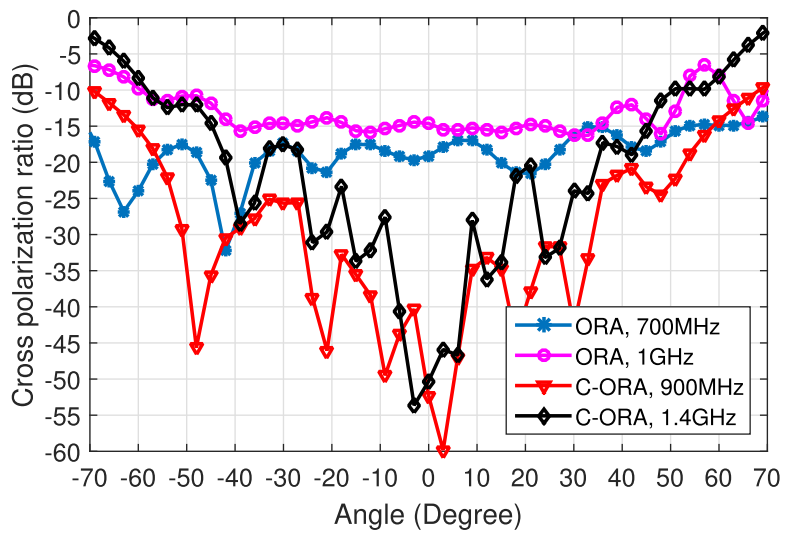

Fig. 11. Cross-polarization ratio performance in the diagonal plane for the C-ORA array, compared with the initial ORA array design.

Expanded polystyrene foam slabs have been used to fill the space between the active layer and the ground plane, and the space between the active and the passive layers. The measured immersed element pattern of the center element in the finite array is compared with the simulated results in Fig. 9. A good agreement is observed in both $\mathrm{E}$ - and H-planes at $900 \mathrm{MHz}$ and $1.4 \mathrm{GHz}$. It confirms that the radiation pattern of a C-ORA element is wide, meeting the requirement of wide-angle scan from the C-ORA array.

An analog beamformer is used to measure the radiation patterns of the subarray with $4 \times 4$ elements at the center of the finite array manufactured. The radiation pattern of the $4 \times 4$ subarray is shown in Fig. 10. Both the main lobe and side lobes from the measurements are in good alignment with the predictions. It is noted that during the pattern measurement, LNAs had been integrated in the subarray of $8 \times 8$. For the patterns from the simulations, no LNA is included. The cross-polarization performance of the original ORA and the $\mathrm{C}$-ORA is compared, recalling the original ORA element is a $L$-shape structure and the C-ORA element is represented by an $X$-shape. The measured cross-polarization ratio for both designs is shown in Fig. 11. The cross-polarization ratio in the $D$-plane is shown to represent the worst case. C-ORA demonstrates an overall better cross-polarization performance than the ORA within the $\pm 45^{\circ}$ scan volume, however, the ORA array has a stable cross polarization variation 
with scan angle for specified frequencies. In both designs, the cross polarization ratio is less than $-15 \mathrm{~dB}$ within the $\pm 45^{\circ}$ scan range.

\section{CONCLUSiON}

A planar array structure is proposed for wideband aperture array design. In this cross ring elements are interconnected by capacitors. The crossed nature of the rings is contrasted with the ORA structure reported earlier. In this paper, the optimization of the ring structure has provided an additional capacitance which combats the dominating inductance at the high-frequency band allowing increased bandwidth. This is especially true at high scan angles, where the necessary capacitance needed cannot be provided by the tip end capacitors alone. A finite array has been manufactured and measured. The results confirm that C-ORA is a good solution to produce wide bandwidth, dual polarizations, and high scan angles while maximizing the element spacing being used.

\section{REFERENCES}

[1] P. N. Wilkinson, "The hydrogen array," in Proc. 131st IAU Colloq. Radio Interferometry, Theory, Techn., Appl., Socorro, NM, USA, vol. 19, T. J. Cornwell and R. A. Perley, Eds. San Francisco, CA, USA: Astronomical Society Pacific, 1991, pp. 428-432.

[2] C. Carilli and S. Rawlings, "Science with the square kilometre array," in New Astronomy Reviews, vol. 48. Amsterdam, The Netherlands: Elsevier, 2004.

[3] A. V. Ardenne, J. D. Bregman, W. A. V. Cappellen, G. W. Kant, and J. G. B. D. Vaate, "Extending the field of view with phased array techniques: Results of European SKA research," Proc. IEEE, vol. 97, no. 8, pp. 1531-1542, Aug. 2009.

[4] G. W. Kant, P. D. Patel, S. J. Wijnholds, M. Ruiter, and E. van der Wal, "EMBRACE: A multi-beam 20,000-element radio astronomical phased array antenna demonstrator," IEEE Trans. Antennas Propag., vol. 59, no. 6, pp. 1990-2003, Jun. 2011.

[5] T. Nakagawa, "Observations of the universe in the terahertz range: From AKARI to SPICA," IEEE Trans. THz Sci. Technol., vol. 5, no. 6, pp. 1133-1139, Nov. 2015.

[6] D. H. Schaubert, A. O. Boryssenko, A. van Ardenne, J. G. B. de Vaate, and C. Craeye, "The square kilometer array (SKA) antenna," in Proc. IEEE Int. Symp. Phased Array Syst. Technol., Oct. 2003, pp. 351-358.

[7] D. H. Schaubert, S. Kasturi, A. O. Boryssenko, and W. M. Elsallal, "Vivaldi antenna arrays for wide bandwidth and electronic scanning," in Proc. 2nd Eur. Conf. Antennas Propag. (EuCAP), Edinburgh, U.K., Nov. 2007, pp. 1-6.

[8] D. H. Schaubert and T. H. Chio, "Wideband vivaldi arrays for large aperture antennas," in Proc. Conf. ASTRON, Dordrecht, The Netherlands, Apr. 1999, p. 49.

[9] H. A. Wheeler, "The radiation resistance of an antenna in an infinite array or waveguide," Proc. IRE, vol. 36, no. 4, pp. 478-487, Apr. 1948.

[10] H. Wheeler, "Simple relations derived fom a phased-array antenna made of an infinite current sheet," IEEE Trans. Antennas Propag., vol. AP-13, no. 4, pp. 506-514, Jul. 1965.

[11] B. A. Munk, Finite Active Arrays. Hoboken, NJ, USA: Wiley, 2003, pp. 136-180. [Online]. Available: http://ieeexplore.ieee.org/xpl/ articleDetails.jsp? arnumber $=5236776$

[12] B. Munk et al., "A low-profile broadband phased array antenna," in Proc. IEEE Antennas Propag. Soc. Int. Symp., vol. 2, Jun. 2003, pp. $448-451$.
[13] B. A. Munk, D. S. Janning, R. J. Marheka, J. F. McCann, and S. W. Schneider, "The design of wideband arrays of closely-spaced wire and slot elements," in Proc. IEEE Antennas Propag. Soc. Int. Symp., Jul. 2010, pp. 1-4.

[14] J. P. Doane, K. Sertel, and J. L. Volakis, "Matching bandwidth limits for arrays backed by a conducting ground plane," IEEE Trans. Antennas Propag., vol. 61, no. 5, pp. 2511-2518, May 2013.

[15] Y. Zhang and A. K. Brown, "Octagonal ring antenna for a compact dual-polarized aperture array," IEEE Trans. Antennas Propag., vol. 59, no. 10 , pp. 3927-3932, Oct. 2011.

[16] Y. Zhang and A. K. Brown, "Design of wide-band dual-polarized aperture array antennas," Int. J. Microw. Wireless Technol., vol. 4, no. 3, pp. 373-378, 2012.

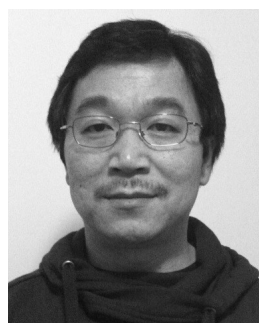

Yongwei Zhang (M'07) received the B.S. degree in communication engineering from Jilin University, Changchun, China, in 1996, and the Ph.D. degree from the Microwave and Communication Systems Research Group, School of Electrical and Electronic Engineering, The University of Manchester, Manchester, U.K., in 2007, with a focus on ultrawideband communications and channel characterization.

In 1996, he joined Lucent Technologies, Qingdao, China, where he was a System Engineer in 5ESS system and Personal Handy-phone System. In 1998, he was a Visitor with the Bell Labs, Naperville, IL, USA, and a Trained Engineer in communication system engineering tools development. From 1996 to 2003, he was with Lucent Technologies, where he was involved in personal-handy-phone system network design and optimization. He was involved in adaptive array antenna design and optimization for wireless communication systems. In 2003, he was with the Microwave and Communication Systems Research Group, The University of Manchester. His current research interests include radio astronomy instrumentations and leading the front-end design work task for the SKA mid-frequency aperture array consortium.

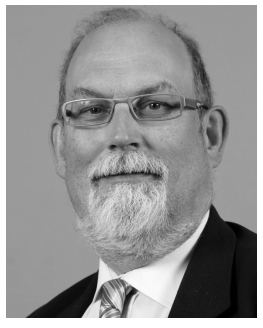

Anthony K. Brown (M'82-SM'06-LSM'18) was the Head of the School of Electrical and Electronic Engineering, The University of Manchester, Manchester, U.K., until 2015, where he was involved in antennas and propagation as applied to radio astronomy instrumentation, wireless communications, and radar. He had a long industrial career with major U.K. companies, where he was involved in radar and satellite communications. In 2003, he joined the School of Electrical and Electronic Engineering. The University of Manchester, where he became the Head of the Microwave and Communications Research Group, and later as the Head of School and an Associate Dean of the Faculty of Science and Engineering. He has been involved in antennas and propagation with over 100 publications on the subject including five patents. He has coedited the book Ultra Wide Band Antennas for Radar and Communications (John Wiley, 2008).

Prof. Brown has been a member of the Square Kilometer Array Radio Telescope U.K. Research Group since 2002 and was a Board Member of the SKA Mid Frequency Array Consortium. He was also a U.K. Representative to the COST ASSIST Action, the Chairman of the Wireless Friendly Building Group, and served on the EUROCAE WG41 Standards Group. He has been involved in computational electromagnetics since the 1980s and in the Applied Computational Electromagnetics Society in 1994. He has been the Chair or Vice Chair of a wide number of international conferences and seminars. He is the Co-Chair of the European Conference on Antennas and Propagation, in 2018. He is a Past Council Member of the Engineering Professors Council and a Fellow of the IET and IMA. He was a Founder Member of the Engineering and Physical Sciences Research Council (U.K.) Communications College. 